\title{
Neutrophil extracellular traps sequester circulating tumor cells and promote metastasis
}

\author{
Jonathan Cools-Lartigue, ${ }^{1}$ Jonathan Spicer, ${ }^{1}$ Braedon McDonald, ${ }^{2}$ Stephen Gowing, \\ Simon Chow, ${ }^{1}$ Betty Giannias, ${ }^{1}$ France Bourdeau, ${ }^{1}$ Paul Kubes, ${ }^{2}$ and Lorenzo Ferri ${ }^{1}$ \\ ${ }^{1}$ LD MacLean Surgical Research Laboratories, Department of Surgery, McGill University, Montreal, Quebec, Canada. \\ ${ }^{2}$ Calvin, Phoebe, and Joan Snyder Institute for Infection, Immunity, and Inflammation, \\ Department of Physiology and Pharmacology, University of Calgary, Calgary, Alberta, Canada.
}

\begin{abstract}
The majority of patients with cancer undergo at least one surgical procedure as part of their treatment. Severe postsurgical infection is associated with adverse oncologic outcomes; however, the mechanisms underlying this phenomenon are unclear. Emerging evidence suggests that neutrophils, which function as the first line of defense during infections, facilitate cancer progression. Neutrophil extracellular traps (NETs) are extracellular neutrophil-derived DNA webs released in response to inflammatory cues that trap and kill invading pathogens. The role of NETs in cancer progression is entirely unknown. We report that circulating tumor cells become trapped within NETs in vitro under static and dynamic conditions. In a murine model of infection using cecal ligation and puncture, we demonstrated microvascular NET deposition and consequent trapping of circulating lung carcinoma cells within DNA webs. NET trapping was associated with increased formation of hepatic micrometastases at $\mathbf{4 8}$ hours and gross metastatic disease burden at $\mathbf{2}$ weeks following tumor cell injection. These effects were abrogated by NET inhibition with DNAse or a neutrophil elastase inhibitor. These findings implicate NETs in the process of cancer metastasis in the context of systemic infection and identify NETs as potential therapeutic targets.
\end{abstract}

\section{Introduction}

Cancer remains a devastating cause of mortality worldwide, with the majority of patients dying as a result of metastasis (1-3). Currently, locoregional control in the form of complete oncologic resection remains an essential curative modality for nearly all solid tumors and provides improved overall and disease-free survival $(2,4,5)$. Control of distant recurrence is predominantly achieved through systemic chemotherapy, with variable results (6-8). However, standard oncologic interventions can have negative consequences. First, manipulation of the primary tumor during surgery is associated with increased numbers of circulating tumor cells (CTCs) (9). Second, infectious complications occur as a result of cancer progression itself, such as bowel obstruction or pneumonia $(10,11)$, and due to complications of standard cancer treatments, such as chemotherapy and surgery (12-14).

Postsurgical infections occur with alarming frequency, with an incidence approaching $40 \%$ in some series (15-17). Given that the majority of the nearly 2 million patients diagnosed with cancer in 2012 in the United States alone underwent at least one surgical procedure, the tremendous potential burden of infection becomes apparent (18). One disturbing feature of severe infectious complications in patients with cancer is their association with adverse oncologic outcomes independent of the morbidity associated with the infectious insult $(14,19-21)$. This phenomenon has been observed across a broad range of malignancies, including lung, esophageal, breast, ovarian, and colorectal cancer, whereby severe

Authorship note: Jonathan Cools-Lartigue and Jonathan Spicer contributed equally to this work and are co-first authors.

Conflict of interest: The authors have declared that no conflict of interest exists. Citation for this article: J Clin Invest. 2013;123(8):3446-3458. doi:10.1172/JCI67484 postoperative infectious complications, such as pneumonia, peritonitis, and sepsis, are significantly associated with an increased rate of death from metastatic disease (21-25).

The cellular players underlying this observation are only beginning to be elucidated. What is becoming apparent is that tumorneutrophil interactions play an important role in linking infection, inflammation, and metastasis $(3,26-31)$. Neutrophils are the predominant circulating granulocyte in humans and comprise $50 \%-75 \%$ of circulating leukocytes (32). They function as the first line of defense against infections, such as those seen in the postoperative period, and are being increasingly recognized as important actors in cancer progression $(3,27,32)$. While antitumor effects have been demonstrated, neutrophils also appear to support the development of metastatic disease under certain conditions $(27,28,30,31)$.

Clinical studies suggest that elevated numbers of circulating neutrophils are an independent marker of adverse prognosis in patients with cancer (26). This has been demonstrated in several malignancies, including lung and gastric cancers $(26,33,34)$. Experimental evidence supports some of these findings. Systemic inflammation induced by lipopolysaccharide administration results in increased adhesion of CTCs within hepatic sinusoids. Neutrophil depletion abolishes this response, which translates into a decrease in overall gross hepatic metastasis formation. This is reversed by polymorphonuclear leukocyte (PMN) reinfusion, providing strong evidence for the involvement of neutrophils in this process. Similarly, neutrophils have been shown to stabilize adhesion of CTCs to pulmonary microvasculature and facilitate gross pulmonary metastasis formation $(3,30)$.

The basic mechanisms by which neutrophils may act to promote the development of metastasis are diverse. Contact-dependent mechanisms, whereby neutrophils act as a bridge, tether- 
A
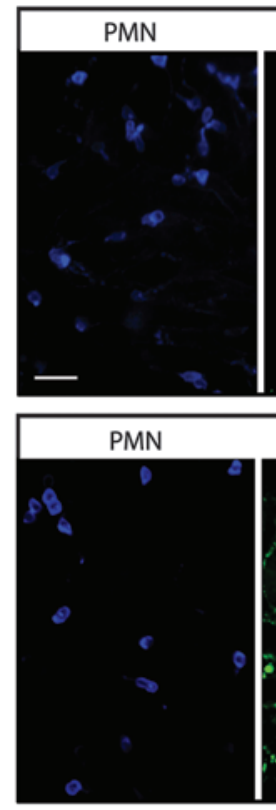

B

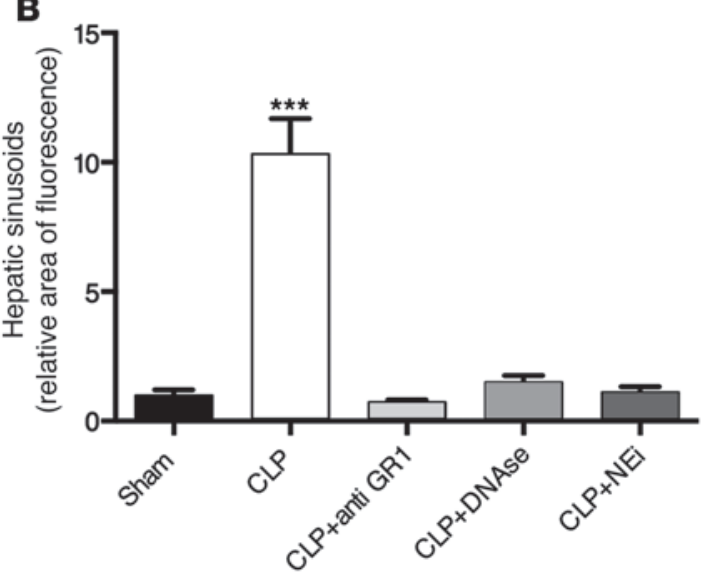

DNA

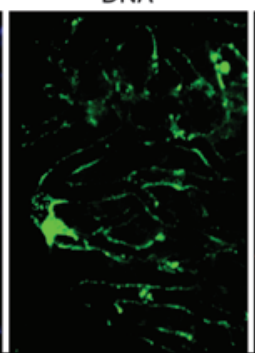

DNA

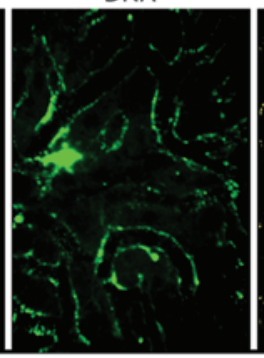

H2AX

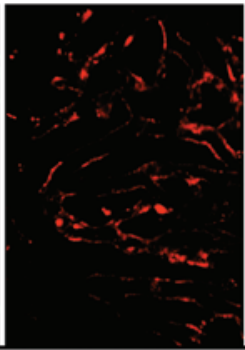

NE

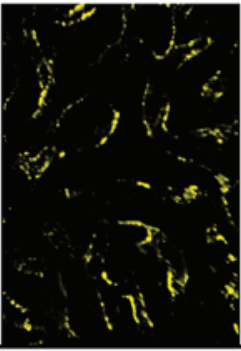

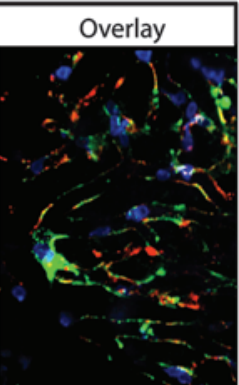

Overlay

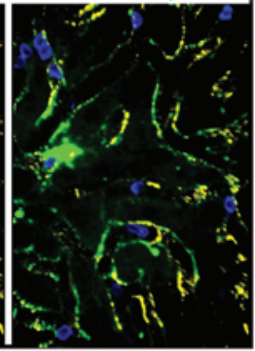

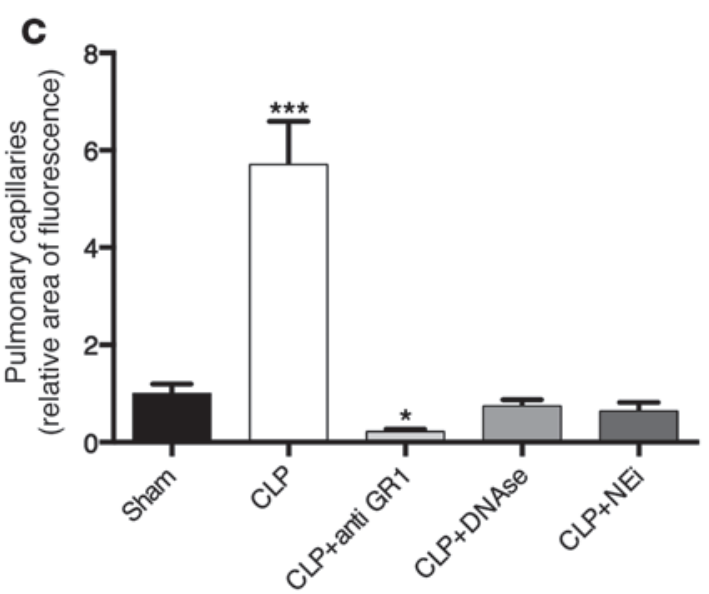

Figure 1

CLP results in widespread deposition of extracellular DNA, which colocalizes with neutrophils and expresses neutrophil-derived granules. (A) Under anesthesia, murine livers were externalized and imaged using SD-IVM, permitting real-time visualization of neutrophil trafficking and DNA extrusion. Intravascular administration of E-fluor 660 anti-GR1, Sytox Green, Alexa Fluor 555 anti-H2AX, or anti-NE was used to visualize polymorphonuclear neutrophils (PMN, blue), DNA (green), H2AX (red), and NE (yellow), respectively. DNA is visualized adjacent to PMN within hepatic sinusoids. DNA stains positive for histone H2AX and NE, in keeping with what has been described for NETs (48). Scale bars: $40 \mu \mathrm{m}$. Extracellular DNA was quantified within (B) hepatic sinusoids and (C) pulmonary capillaries by measuring total area of fluorescence per hpf over $5 \mathrm{hpf}$. Data are represented as relative area of fluorescence compared with sham. CLP was associated with increased amounts of extracellular DNA compared with sham. Neutrophil depletion or systemic administration of DNAse 1 or NEi after CLP results in decreased extracellular DNA staining compared with CLP alone. Data are presented as mean \pm SEM from $n=3-5$ mice per group. ${ }^{* * *} P<0.001,{ }^{*} P<0.05$ versus sham as determined by 1-way ANOVA with Tukey's HSD post-hoc analysis. See also Supplemental Figures 1 and 2.

ing CTCs to end-organ endothelium, have been described (19). This interaction is mediated by the interaction of $\beta_{2}$ integrins on neutrophils and ICAM-1 on tumor cells (31,35-37). Additional contact-independent mechanisms have also been proposed. Neutrophils are able to secrete soluble factors that can activate endothelium and parenchymal cells, enhancing CTC adhesion in distant sites $(27,38-40)$.

In addition to the mechanisms proposed thus far, novel aspects of neutrophil biology may contribute to cancer progression and metastasis. Neutrophil extracellular traps (NETs) are neu- trophil-derived structures composed of extruded DNA, decorated with antimicrobial proteins (41-45). NETs are formed in response to infectious stimuli and serve as a host defense against pathogens, trapping and killing bacterial, fungal, and protozoan invaders in vitro $(41,46,47)$. In addition, NETs have been shown to effectively trap circulating bacteria within the liver in experimental models of sepsis (48). In contrast to their postulated beneficial role, exaggerated NET formation is implicated in a number of pathologies, including the multiorgan dysfunction that may accompany sepsis $(12,49,50)$. Given the potential association between severe infec- 
A
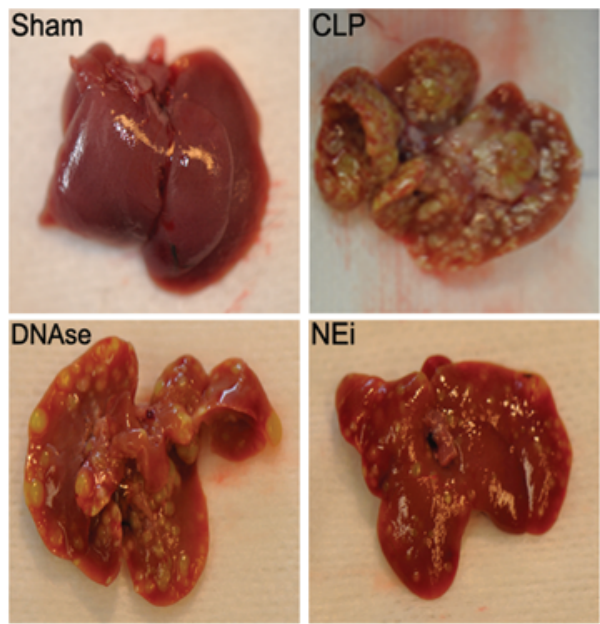

B

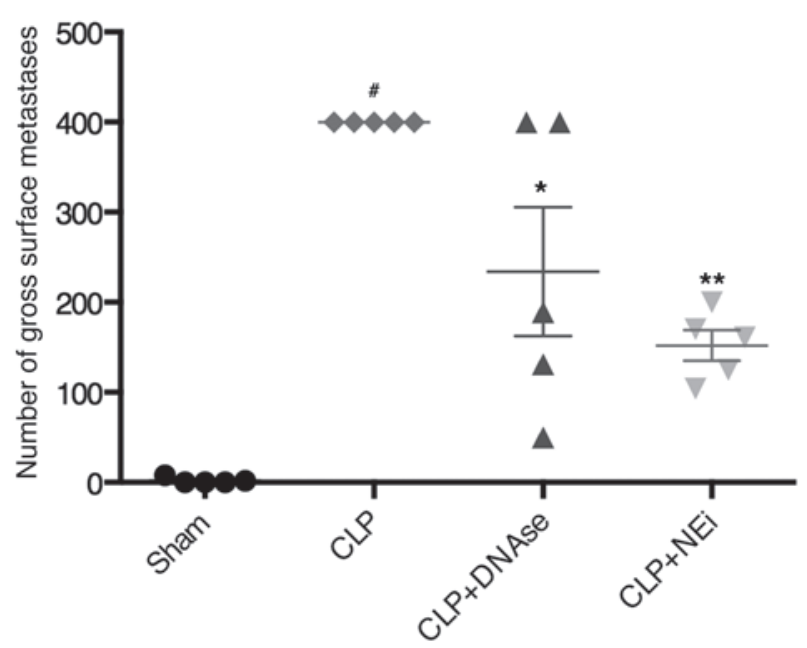

tion and tumor progression, we sought to determine whether a role for NETs exists in a model of severe postoperative infection. We hypothesize that, in the context of severe infection, NETs are able to trap CTCs, thus promoting early adhesion of tumor cells to distant organ sites. Furthermore, by promoting early adhesive events, NETs may facilitate metastatic disease progression.

\section{Results}

Sepsis induced by cecal ligation and puncture results in widespread deposition of NETs. In the setting of disseminated infection, bacteremia, and sepsis, neutrophils demonstrate NET formation in vivo (48). Cecal ligation and puncture (CLP) invariably results in polymicrobial abdominal sepsis and systemic sepsis, which are characteristic features of postoperative infections following gastrointestinal surgery in humans. Accordingly, it was selected in lieu of systemic administration of individual bacterial components, such as LPS, to induce systemic infection $(51,52)$. In order to determine whether CLP induces NET formation, multichannel spinning disk confocal intravital microscopy (SD-IVM) was used to visualize hepatic and pulmonary microvasculature within mice subjected to CLP (Figure 1 and Supplemental Figure 1; supplemental material available online with this article; doi:10.1172/JCI67484DS1).

\section{Figure 2}

Systemic sepsis promotes the development of gross metastasis, which is attenuated by systemic administration of inhibitors of NET formation. Mice were subjected to CLP in order to induce sepsis. Intrasplenic injection of H59 Lewis lung cancer cell lines was performed 24 hours later. Administration of DNAse 1 intramuscularly or a NEi per os was started 24 hours prior to CLP and continued daily for 14 days. At 14 days, mice were sacrificed, and the number of gross hepatic metastases was quantified. (A) Representative images of hepatic nodules after necropsy in mice subjected to sham surgery, CLP, CLP with daily DNAse 1 administration, and CLP with daily NEi administration. (B) CLP resulted in a significant increase in the number of gross metastatic nodules compared with sham. Treatment with DNAse or $\mathrm{NEi}$ after CLP resulted in a significant decrease in the number of gross metastases. Data are presented as mean \pm SEM from $n=5$ mice per group. ${ }^{*} P<0.05,{ }^{*} P<0.01$ versus CLP. ${ }^{\sharp} P<0.001$ versus sham as determined by 1-way ANOVA with Tukey's HSD post-hoc analysis.

NETs are composed of extracellular DNA decorated with neutrophil-derived granules and modified histones (48). Visualization of extracellular DNA was performed by intravascular injection of the cell impermeable dye, Sytox Green. This revealed web-like DNA within hepatic sinusoidal spaces and pulmonary capillaries (Figure 1 and Supplemental Figure 1). To further support the claim that these extracellular webs represent NETs, we demonstrated colocalization of DNA with neutrophil-derived granules. This was achieved by intravascular administration of fluorescently labeled antibodies against polymorphonuclear neutrophils (PMN efluor 660 anti-GR1), histone H2AX (Alexa Fluor 555 anti-H2AX), neutrophil elastase (NE) (Alexa Fluor 555 anti-NE), and Sytox Green (Figure 1A and Supplemental Figure 1).

Quantification of microvascular DNA was subsequently performed in mice subjected to CLP, in mice subjected to neutrophil depletion 24 hours prior to CLP, and in mice administered DNAse 1 or NE inhibitor (NEi) after CLP. Relative area of fluorescence was determined in each high-power field (hpf) of view, expressed relative to mice subjected to sham surgery alone (relative area of fluorescence $=1$; Figure 1, B and C). Significantly more microvascular Sytox Green staining was observed in mice subjected to CLP compared with mice subjected to sham surgery (10.3 and 5.7 relative area of fluorescence in liver and lungs, respectively; $P<0.001$ compared with sham). Neutrophil depletion ( 0.74 and 0.2 relative area of fluorescence in liver and lungs, respectively; $P=\mathrm{NS} ; P<0.05$ compared with sham, respectively) or systemic administration of DNAse 1 (1.52 and 0.74 relative area of fluorescence in liver and lungs, respectively; $P=$ NS compared with sham) or NEi (1.13 and 0.63 relative area of fluorescence in liver and lungs, respectively; $P=$ NS compared with sham) after CLP was associated with levels of NET deposition comparable to those after sham surgery (Figure 1, B and C, and Supplemental Figure 2).

Systemic sepsis promotes the development of gross metastasis, which is attenuated by systemic administration of inhibitors of NET formation. Having shown that CLP-induced sepsis results in widespread microvascular NET deposition, we sought to establish an association between NET formation and metastasis. Twenty-four hours after performing CLP or sham surgery, H59 Lewis lung carcinoma cells (referred to as H59 cells hereafter) were injected via the intrasplenic route. CLP animals were either left untreated or received DNAse $1(2.5 \mathrm{mg} / \mathrm{kg})$ intramuscularly or a NEi per os daily $(2.2 \mathrm{mg} / \mathrm{kg})$. Both of these reagents are known inhibitors of NET formation and were used to determine whether disrupting 
A
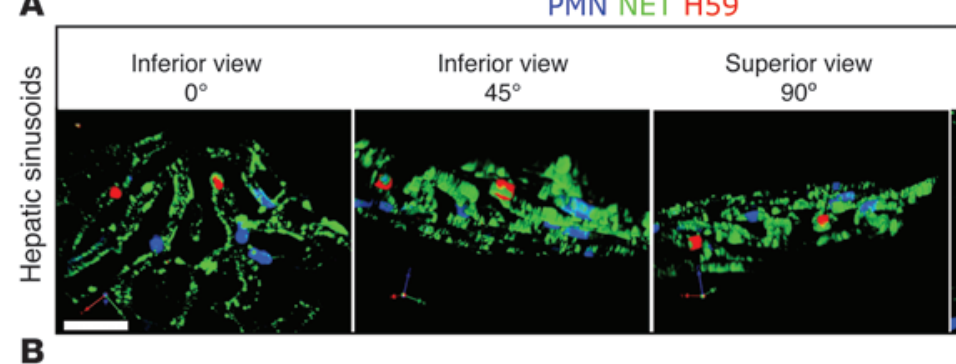

$90^{\circ}$

B
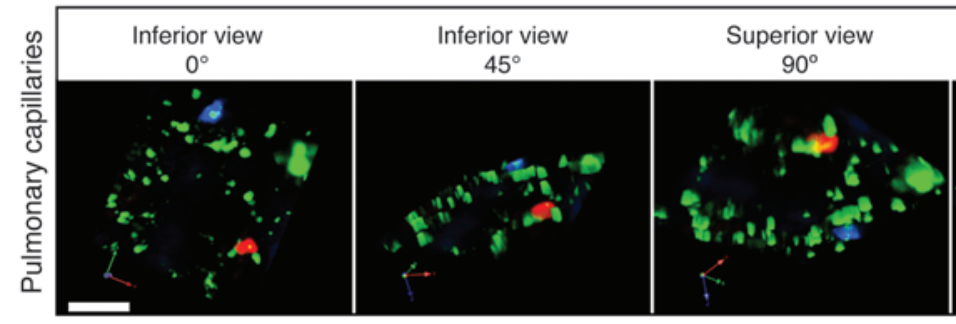

\section{c}
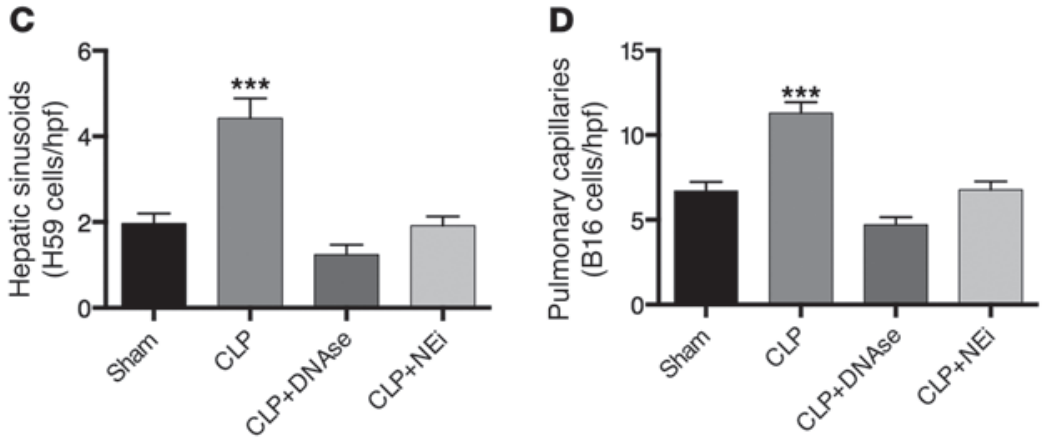

Figure 3

Tumor cell adhesion after CLP is augmented by trapping within neutrophil-derived extracellular DNA. In order to demonstrate that tumor cells become embedded within NETs, SD-IVM was performed permitting visualization of $(\mathbf{A})$ hepatic sinusoids in living mice and $(\mathbf{B})$ pulmonary capillaries ex vivo within 10 minutes. Images shown in A and $\mathbf{B}$ represent a single 3-dimensional reconstruction of confocal z-stacks (10- to $20-\mu \mathrm{m}$ thickness, $1-\mu \mathrm{m}$ intervals), rotated $180^{\circ}$ from an inferior to a superior perspective. In both liver and lungs, tumor cells (red) were found to arrest within extracellular chromatin (Alexa Fluor 555 anti-histone H2AX [green]) adjacent to neutrophils (E-fluor 660 anti-GR1 [blue]). Scale bars: $20 \mu \mathrm{m}$. See also Supplemental Video 1 and Supplemental Figure 3. (C) Quantification of adhesion of H59 cells within hepatic sinusoids. $3 \times 10^{4}$ cells were injected via the spleen 24 hours after CLP or sham surgery. Adhesion was increased after CLP compared with sham. Systemic administration of DNAse 1 or NEi starting 1 day prior to CLP abolished this increase. (D) Quantification of B16 melanoma cells within pulmonary capillaries. $1 \times 10^{6}$ cells were injected via tail vein 24 hours after CLP or sham. Adhesion was increased after CLP compared with sham and was abolished by administration of DNAse 1 or NEi starting 1 day prior to CLP. Quantification was performed by counting the number of cells per hpf in 8 to $10 \mathrm{hpf}(\times 20)$ per experiment. Data are presented as mean \pm SEM from $n=5$ mice per group. ${ }^{* \star *} P<0.001$ versus sham, DNAse, and NEi as determined by 1-way ANOVA with Tukey's HSD post-hoc analysis.

NET formation would alter the course of hepatic tumor progression $(53,54)$. Quantification of gross hepatic metastases was performed at necropsy at 2 weeks (Figure 2). Significantly more gross hepatic metastases were observed in CLP mice compared with mice after sham surgery (mean 400 nodules versus 2 nodules; $P<0.0001$ ) (Figure 2B). Systemic administration of DNAse 1 or NEi after CLP attenuated the development of hepatic metastases at 2 weeks compared with administration of CLP alone (mean 234 and 132 nodules, respectively; $P<0.05$ and $P<0.01$ ) (Figure $2 \mathrm{~B}$ ).

NET deposition results in increased tumor cell adhesion to hepatic and pulmonary microvasculature in vivo. Early adhesive events play an important role in the development of metastasis $(3,30)$. Hav-
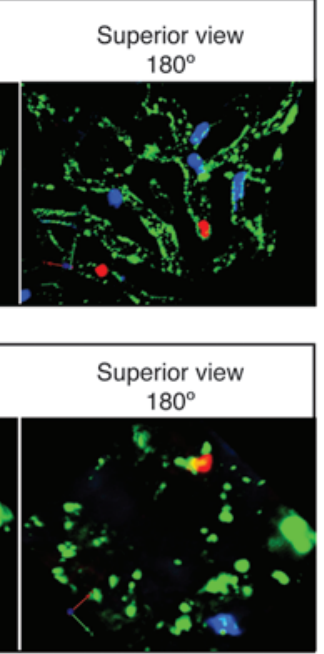

ing shown that CLP induces NET formation and that manipulating expression of NETs with DNAse and NEi attenuates metastasis formation, the possibility that NETs facilitate metastasis through CTC trapping arises. In an attempt to visualize real-time tumor-NET interactions in vivo, SD-IVM was used (Figure 3). Intravascular injection of fluorescently labeled antibodies against histone H2AX (green) and GR1 (blue) was used to visualize NETs and neutrophils, respectively. Histone $\mathrm{H} 2 \mathrm{AX}$ has been shown to provide stable and robust staining of NETs in vivo (48). Following intravascular injection of H59 cells (red), colocalization within areas of histone staining and neutrophils was observed in both hepatic and pulmonary microvasculature (Figure 3, $A$ and B, respectively, and Supplemental Figure 3). Real-time video acquisition demonstrated arrest of tumor cell movement within areas of dense histone staining. Conversely, neutrophil locomotion through hepatic sinusoids was not impeded, suggesting that tumor cell arrest was not due to sinusoidal plugging (Supplemental Video 1).

Epifluorescent intravital microscopy was used to quantify tumor cell adhesion within hepatic sinusoids or pulmonary capillaries following intravascular injection of H59 or B16-F10 melanoma cells (referred to as B16 cells hereafter), respectively (Figure 3, C and D). Two cell lines and two organ sites were assessed in order to support our hypothesis that NET-mediated cell trapping is neither cell nor organ specific. Twenty-four hours following CLP, intrasplenic injection of H59 cells was performed. Adhesion was quantified 10 minutes later by counting the number of cells in 8 to 10 random hpf. Tumor cell sinusoidal arrest was significantly increased after CLP compared with that in mice subjected to sham surgery or those administered DNAse 1 or NEi after CLP (mean 4.5 cells per hpf versus $1.9,1.2$, and 1.9 cells per hpf, respectively; $P<0.001$ ) (Figure 3C). Similarly, after tail vein injection of B16 cells, increased tumor cell arrest within pulmonary capillaries was observed after CLP compared with that in mice subjected to sham surgery or those administered DNAse 1 or NEi after CLP (mean 11.7 cells per hpf versus 6.7, 4.7, and 6.7 cells per hpf, respectively; $P<0.001$ ) (Figure $3 \mathrm{D}$ ). 
A

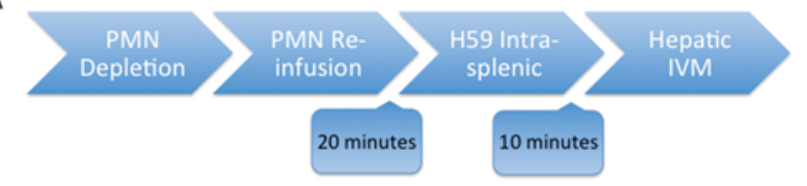

\begin{tabular}{|c|c|c|c|c|c|}
\hline & Control & PMN & PMN+PMA & $\begin{array}{l}\text { PMN+PMA } \\
\text { +DNAse }\end{array}$ & $\begin{array}{c}\text { PMN+PMA } \\
+ \text { +NEi }\end{array}$ \\
\hline Step 1 & PMN - & PMN - & PMN - & PMN - & PMN - \\
\hline Step 2 & 500nM PMA & $1 \times 10^{6} \mathrm{PMN}$ & $\begin{array}{l}1 \times 10^{6} \text { PMN + } \\
500 \mathrm{nM} \text { PMA }\end{array}$ & $\begin{array}{c}1 \times 10^{6} \text { PMN + } \\
500 \mathrm{nM} \text { PMA } \\
+1000 \mathrm{U} \\
\text { DNAse } 1\end{array}$ & $\begin{array}{l}1 \times 10^{6} \text { PMN + } \\
500 \mathrm{nM} \text { PMA } \\
+10 \mu \mathrm{M} \text { NEi }\end{array}$ \\
\hline Step 3 & $\begin{array}{c}5 \times 10^{4} \text { H59. } \\
\text { GFP }\end{array}$ & $\begin{array}{c}5 \times 10^{4} \text { H59- } \\
\text { GFP }\end{array}$ & $\begin{array}{c}5 \times 10^{4} \mathrm{H} 59- \\
\text { GFP }\end{array}$ & $\begin{array}{c}5 \times 10^{4} \mathrm{H} 59- \\
\text { GFP }\end{array}$ & $\begin{array}{c}5 \times 10^{4} \text { H59- } \\
\text { GFP }\end{array}$ \\
\hline
\end{tabular}

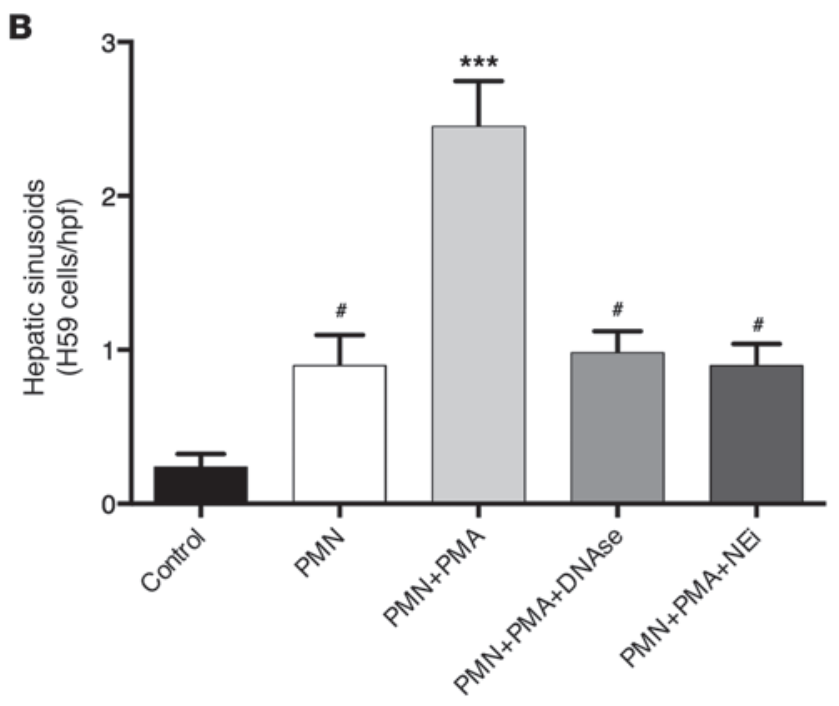

CLP is a potent inflammatory stimulus, which may exert numerous systemic effects extending beyond NET formation $(51,52)$. Accordingly, we sought to determine whether systemic LPS administration, an established model of microvascular NET deposition in vivo, could produce comparable levels of tumor cell adhesion to those after CLP administration (43). Quantification of H59 cells within hepatic sinusoids 4 hours after intraperitoneal LPS administration yielded comparable results. LPS injection resulted in significantly increased tumor cell adhesion to hepatic sinusoids compared with controls (mean 2.34 cells per hpf versus 0.42 cells per hpf, respectively; $P<0.001)$. Systemic administration of DNAse or NEi in mice subjected to LPS stimulation resulted in levels of tumor cell adhesion comparable to those of mice after sham surgery (mean 0.37 cells per hpf and 0.55 cells per hpf; $P=$ NS) (Supplemental Figure 4).

NET formation in the absence of systemic inflammation is sufficient to increase tumor adhesion in vivo. While the data thus far demonstrate an association between NET formation and CTC arrest, the relationship remains circumstantial. We therefore sought to clarify whether NETs themselves enhance tumor cell arrest in the context of systemic sepsis or if their presence is merely concomitant but noncontributory. Because systemic inflammation induces a multitude of effects on numerous cell types, which may contribute to tumor adhesion, mice were not subjected to any overt inflammatory stimuli. Furthermore, in an attempt to limit the effects of our interventions (Figure 4A) to predominantly neutrophils, all mice

\section{Figure 4}

NET production by neutrophils is sufficient to increase tumor cell adhesion within hepatic sinusoids. (A) A schematic representation of the experimental design is depicted. All mice used were subjected to neutrophil depletion via intraperitoneal injection of anti-GR1 $(150 \mu \mathrm{g})$. Twenty-four hours later, $1 \times 10^{6}$ bone marrow-derived neutrophils (or control buffer) from syngeneic mice were reinfused via the intrasplenic route. This was followed by injection of $3 \times 10^{4} \mathrm{H} 59$-GFP cells into the spleen 20 minutes later. Ten minutes later, epifluorescence microscopy was used for quantification of adherent cells. IVM, intravital microscopy. (B) Quantification of arrested tumor cells within hepatic sinusoids $10 \mathrm{~min}-$ utes following intrasplenic injection of H59-GFP cells. Injection of $500 \mathrm{nM}$ PMA without neutrophils prior to tumor cell injection resulted in low levels of tumor cell adhesion. Infusion of unstimulated neutrophils increased tumor cell adhesion above control, but this was significantly lower than when neutrophils were pretreated with $500 \mathrm{nM}$ PMA. This increase is abrogated if PMA treatment of neutrophils occurs in the presence of $\mathrm{NEi}$ $(10 \mu \mathrm{m})$ or DNAse $1(1,000 \mathrm{U})$ prior to reinfusion. Adherent cells were quantified by counting the number of cells per hpf in 8 to $10 \mathrm{hpf}(\times 20)$ per experiment. Data are presented as mean \pm SEM from $n=4-5$ mice per group. Significance was determined using 1-way ANOVA with Tukey's HSD post-hoc analysis. ${ }^{* \star *} P<0.0001,{ }^{\#} P<0.05$ compared with control.

were subjected to neutrophil depletion, followed by reinfusion of bone marrow-derived neutrophils from syngeneic mice. Following neutrophil reinfusion, $\mathrm{H} 59$ cells were injected via the intrasplenic route, and their adhesion was quantified within hepatic sinusoids using epifluorescence in vivo microscopy (Figure 4B). Inoculation of unstimulated neutrophils followed by H59 cells resulted in minimal tumor cell adhesion (0.9 cells per hpf). Stimulation of neutrophils with $500 \mathrm{nM}$ PMA to induce NET formation prior to inoculation of $\mathrm{H} 59$ cells resulted in a nearly 3 -fold increase in tumor cell adhesion ( 2.45 cells per hpf; $P<0.0001$ versus unstimulated neutrophils alone), which was abolished if PMA-stimulated neutrophils were treated with either $10 \mu \mathrm{M}$ NEi or $1,000 \mathrm{U}$ DNAse 1 ( 0.9 and 0.98 cells per hpf, respectively; $P=$ NS versus unstimulated neutrophils). Intrasplenic injection of tumor cells without neutrophil reinfusion, even in the presence of $500 \mathrm{nM}$ PMA, resulted in near negligible sinusoidal arrest ( 0.2 cells per hpf; $P<0.001$ versus unstimulated neutrophils), suggesting that the stimulatory effects of PMA alone were not responsible for the observed adhesive phenotype. Thus, while intact NETs do not appear to be critical for tumor cell adhesion to take place, their presence appears to significantly augment this process.

NET deposition results in increased tumor cell adhesion in vitro. In order to support the in vivo observation that tumor cells can become trapped within NETs, we sought to demonstrate this phenomenon in vitro in a system using only tumor cells and neutrophils. PMA (800 nM) was used to simulate NET formation in neutrophil monolayers. Fluorescently labeled murine (H59) or human (A549) lung cancer cells were allowed to incubate with neutrophils in order for adhesion to occur. After washing, adhesion was quantified as the number of cells per hpf averaged across 5 fields. Adhesion of H59 or A549 cells to neutrophil monolayers was significantly increased after PMA stimulation compared with control (mean $14 \mathrm{H} 59$ and 21.4 A549 cells per hpf versus $3.2 \mathrm{H} 59$ and 4.1 A549 cells per hpf; $P<0.001$ ) (Figure 5A). Conversely, degradation of NETs using DNAse $1(1,000 \mathrm{U})$ or inhibition of NET formation by pretreatment of neutrophil monolayers with NEi $(5 \mu \mathrm{M})$ abrogated the increased tumor adhesion observed after PMA stimulation (mean 4.6 H59 and 8.7 A549 cells per 

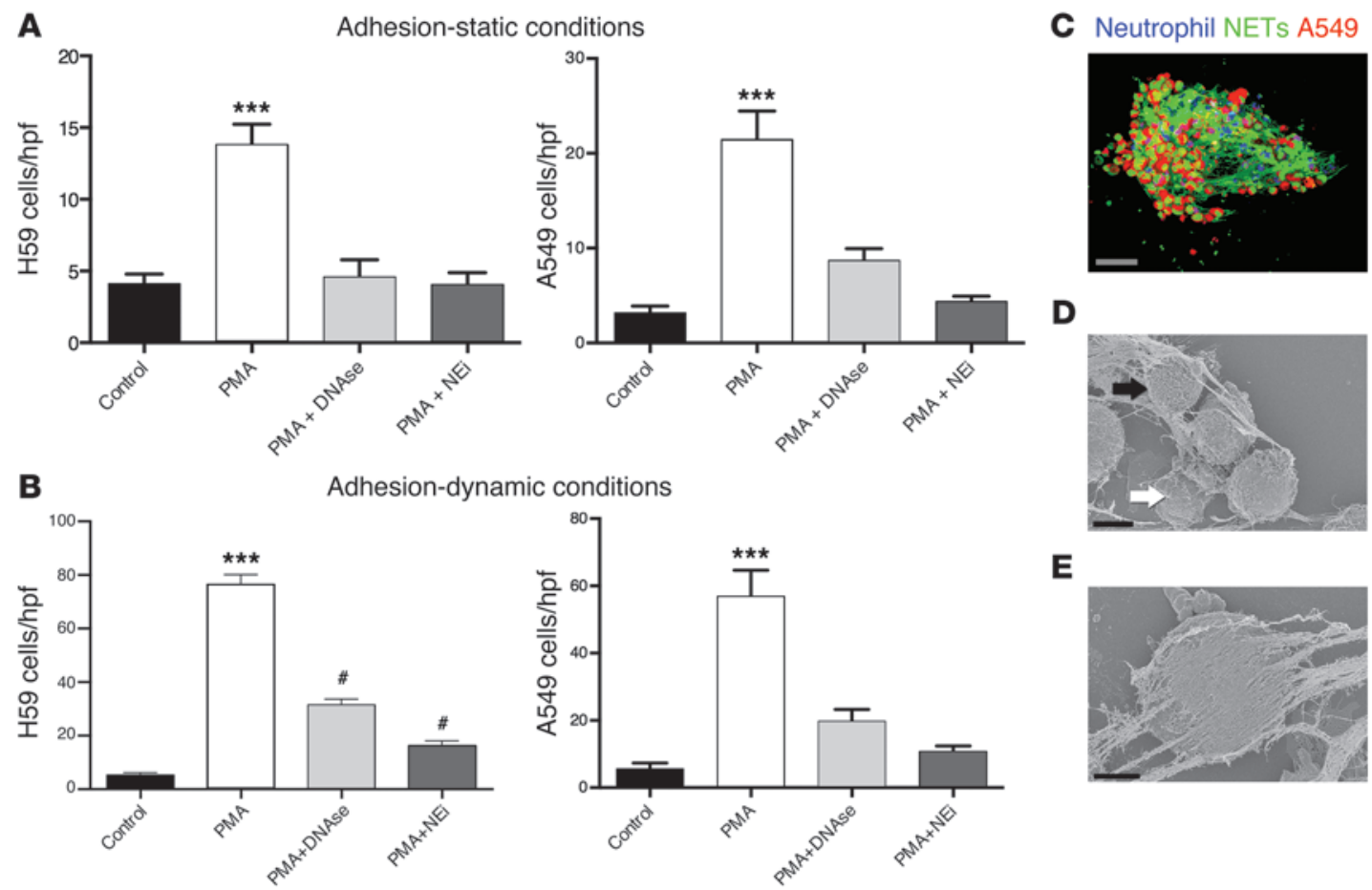

$\mathbf{E}$

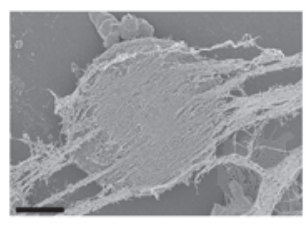

\section{Figure 5}

NETs trap both human and murine tumor cells in vitro. (A) Under static conditions, H59 and A549 cells demonstrate increased adhesion to neutrophil monolayers stimulated with PMA $(800 \mathrm{nM})$ compared with unstimulated neutrophils. Addition of DNAse 1 (1,000 U) or pretreatment of neutrophils with NEi $(5 \mu \mathrm{M})$ results in levels of adhesion comparable to control. (B) Tumor cells were perfused over neutrophil monolayers at shear rates of 1 dyne/cm/s-1. $\mathrm{H} 59$ and A549 cells demonstrate increased adhesion to neutrophils after stimulation with PMA (800 nM) compared with controls. This was abrogated by addition of DNAse $1(1,000 \mathrm{U})$ or pretreatment of neutrophils with NEi $(5 \mu \mathrm{M})$. Data are presented as mean $\pm \mathrm{SEM}$ from $n=2-4$ separate experiments. ${ }^{* \star \star} P<0.01$ versus control, DNAse, and NEi. ${ }^{*} P<0.05$ versus control. Significance was determined using 1-way ANOVA with Tukey's HSD post-hoc analysis. (C) Confocal imaging reveals that after PMA stimulation, A549 cells (red) become trapped within webs of extracellular DNA (green) in proximity to neutrophils (blue). (D) Scanning electron microscopy of A549- and PMA-stimulated neutrophils $(800 \mathrm{nM})$ (original magnification, $\times 3,000)$. After PMA stimulation, neutrophils flatten (white arrow) and extrude strands consistent with NETs. Strands encompass a cluster of adherent tumor cells (black arrow). (E) Scanning electron microscopy demonstrates that NETs are in direct contact with adherent A549 tumor cells (original magnification, $\times 3,500$ ). Scale bars: $40 \mu \mathrm{m}$ (confocal microscopy); $5 \mu \mathrm{m}$ (electron microscopy). See also Supplemental Figures 2 and 3.

hpf after DNAse 1, $4.1 \mathrm{H} 59$ and 4.4 A549 cells per hpf after NEi; $P<0.001$ versus PMA alone) (Figure 5A). This was observed in both cell lines, arguing against a cell-specific phenomenon.

In order to more closely approximate physiologic conditions, we sought to determine whether tumor cell adhesion to NETs is maintained under conditions of flow. This was achieved using a parallel-plate flow chamber, in which H59 or A549 cells were perfused over neutrophil monolayers at a rate of $1 \mathrm{dyne} / \mathrm{cm} / \mathrm{s}^{-1}$. Addition of PMA $(800 \mathrm{nM})$ to the tumor cell perfusate resulted in rapid formation of NETs, resulting in a significant increase in tumor cell adhesion compared with control (mean 75.6 H59 and 56.9 A549 cells per hpf versus 5.4 H59 and 5.7 A549 cells per hpf; $P<0.001$ ) (Figure 5B). Adhesion was significantly reduced by addition of DNAse $1(1,000 \mathrm{U})$ to the perfusate or by pretreatment of neutrophils with NEi $(5 \mu \mathrm{M})$ (mean 31.5 H59 and 19.8 A549 cells per hpf after DNAse 1, $16.3 \mathrm{H} 59$ and 10.8 A549 cells per hpf after NEi; $P<0.001$ versus PMA alone) (Figure 5B).

Real-time video acquisition of $\mathrm{H} 59$ cells perfused over neutrophil monolayers was performed in order to visualize tumor cell entrapment within NETs. Addition of $800 \mathrm{nM}$ PMA to the tumor cell perfusate resulted in the rapid extrusion of NETs, as visualized by
Sytox staining. Extrusion of extracellular DNA was accompanied by the rapid arrest of tumor cells. Addition of 1,000 U DNAse 1 to the perfusate resulted in a dramatic detachment of adherent cells in conjunction with a loss of extracellular DNA (data not shown).

Tumor cell-NET interactions in vitro were visualized using confocal microscopy (Figure 5C). After PMA stimulation of neutrophil monolayers, clusters of A549 cells were visualized within webs of extracellular DNA. Neutrophils were visualized within tumorDNA clusters but were not in direct contact with malignant cells, suggesting the adhesive mechanism was mediated by trapping within NETs. Scanning electron microscopy was performed in order to more closely visualize the interaction between NETs and tumor cells (Figure 5, D and E). After PMA stimulation, neutrophil morphology was characterized by a flattening of the plasma membrane with extrusion of fibrillar material consistent with NETs in the extracellular environment (Supplemental Figure 5). NETs appeared to be wrapped around adherent A549 cells and were visualized in direct contact with the tumor cell membrane.

The effects of PMA, DNAse 1, and NEi on NET formation were subsequently confirmed in vitro. Treatment of neutrophils with either DNAse 1 or NEi resulted in profound disruption of 
A

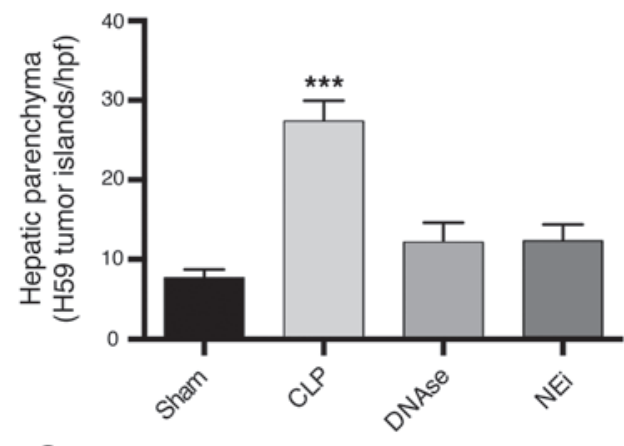

C

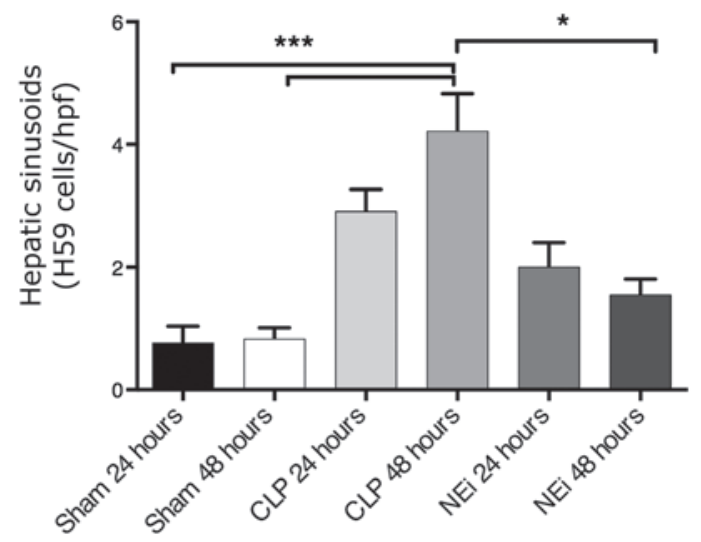

B

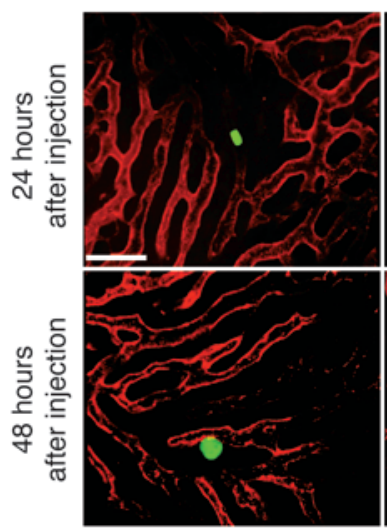

Sham
H59 Hepatic sinusoids

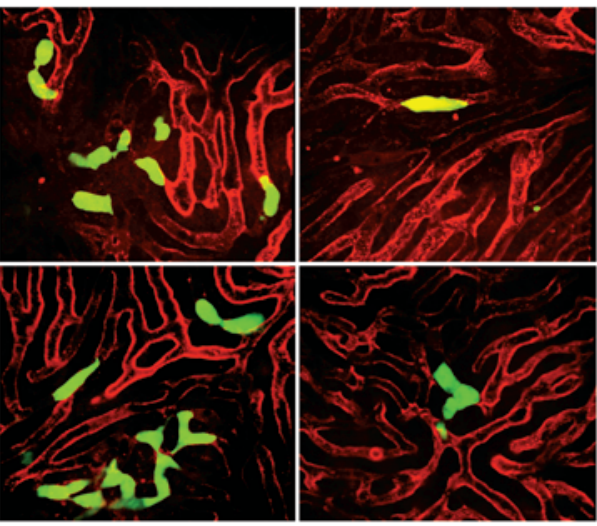

$\mathrm{NEi}$

\section{Figure 6}

CLP promotes the development of micrometastases, which is attenuated by treatment with DNAse 1 and NEi. H59 cells were injected via the spleen 24 hours after CLP. (A) Quantification of micrometastatic foci demonstrated increased numbers of tumor islands at 48 hours after intrasplenic injection in mice subjected to CLP compared with those after sham surgery. Systemic administration of DNAse 1 or NEi after CLP is associated with decreased micrometastases compared with CLP alone. (B) Representative images of tumor cells trapped within hepatic sinusoidal spaces in mice subjected to sham surgery, CLP, and NEi after CLP at 24 and 48 hours following intrasplenic injection of $5 \times 10^{5} \mathrm{H} 59$ cells. Scale bars: $40 \mu \mathrm{m}$. (C) To further demonstrate that adherent tumor cells persist and are able to grow in this model of sepsis, individual tumor cells (as depicted in B) were quantified within the liver at 24 and 48 hours after intrasplenic injection. Quantification of individual tumor cells within hepatic sinusoids reveals increased numbers at both 24 and 48 hours after intrasplenic injection in mice subjected to CLP compared with sham. Systemic administration of NEi after CLP resulted in decreased numbers of tumor cells at both 24 and 48 hours compared with CLP alone. Data were acquired using $n=3-5$ mice per group. Quantification was performed by counting the number of cells per 5-10 hpf per experiment $(\times 20) .{ }^{*} P<0.05$, ${ }^{* *} P<0.0001$ compared with control, DNAse, and sham. Significance was determined using 1-way ANOVA with Tukey’s HSD post-hoc analysis.

extracellular DNA or inhibition of NET extrusion, as previously described (Supplemental Figure 6, A and B, and refs. 53, 54). To ensure that the differences in adhesion were not due to differences in neutrophil numbers after treatment with DNAse 1 or NEi, this was quantified directly. PMA stimulation increased adhesion of neutrophils to coverslips. However, numbers were unaffected after treatment with DNAse 1 or NEi (Supplemental Figure 6C).

Tumor cell trapping within NETs is associated with increased micrometastases at 48 hours. We have previously shown that early adhesive events are important in the development of gross metastatic disease (30). In the context of this study, we sought to determine whether the increased adhesion observed in animals with CLP-induced sepsis translated into a greater number of micrometastases (Figure 6). Epifluorescent microscopy was used in order to quantify the number of hepatic tumor islands 48 hours after intrasplenic H59 injection. The number of tumor islands visualized was significantly higher in mice after CLP compared with that in mice after sham surgery (mean 27.4 tumor islands per hpf versus 7.7 tumor islands per hpf, respectively; $P<0.0001)$. In keeping with our results thus far, systemic administra- tion of DNAse or NEi after CLP abrogated micrometastatic tumor formation to levels comparable to those after sham surgery (mean 12.16 and 12.31 tumor islands per hpf versus 7.7 tumor islands per hpf, respectively; $P=\mathrm{NS}$ ). Taken together, these results suggest that tumor cell entrapment within NETs promotes stable retention, permitting subsequent growth within the liver (Figure 6A).

In order for micrometastatic foci to form, adherent cells must persist within the microvasculature. In an attempt to demonstrate this, SD-IVM was used to quantify the number of individual adherent $\mathrm{H} 59$ cells within the liver at 24 and 48 hours after intrasplenic injection (Figure 6, B and C). In mice subjected to sham surgery, few entrapped cells were visualized at 24 hours and no increased number in adherent cells was observed at 48 hours (mean 0.76 cells per hpf at 24 hours, 0.82 cells per hpf at 48 hours; $P=$ NS) (Figure 6C). Conversely, in CLP-treated mice, significantly more H59 cells remained adherent within the liver, a trend that persisted at 48 hours (mean 2.9 cells per hpf at 24 hours and 4.2 cells per hpf at 48 hours versus sham; $P<0.001$ ) (Figure 6C). In mice systemically administered NEi after CLP, no difference in the number of cells persisting within the 

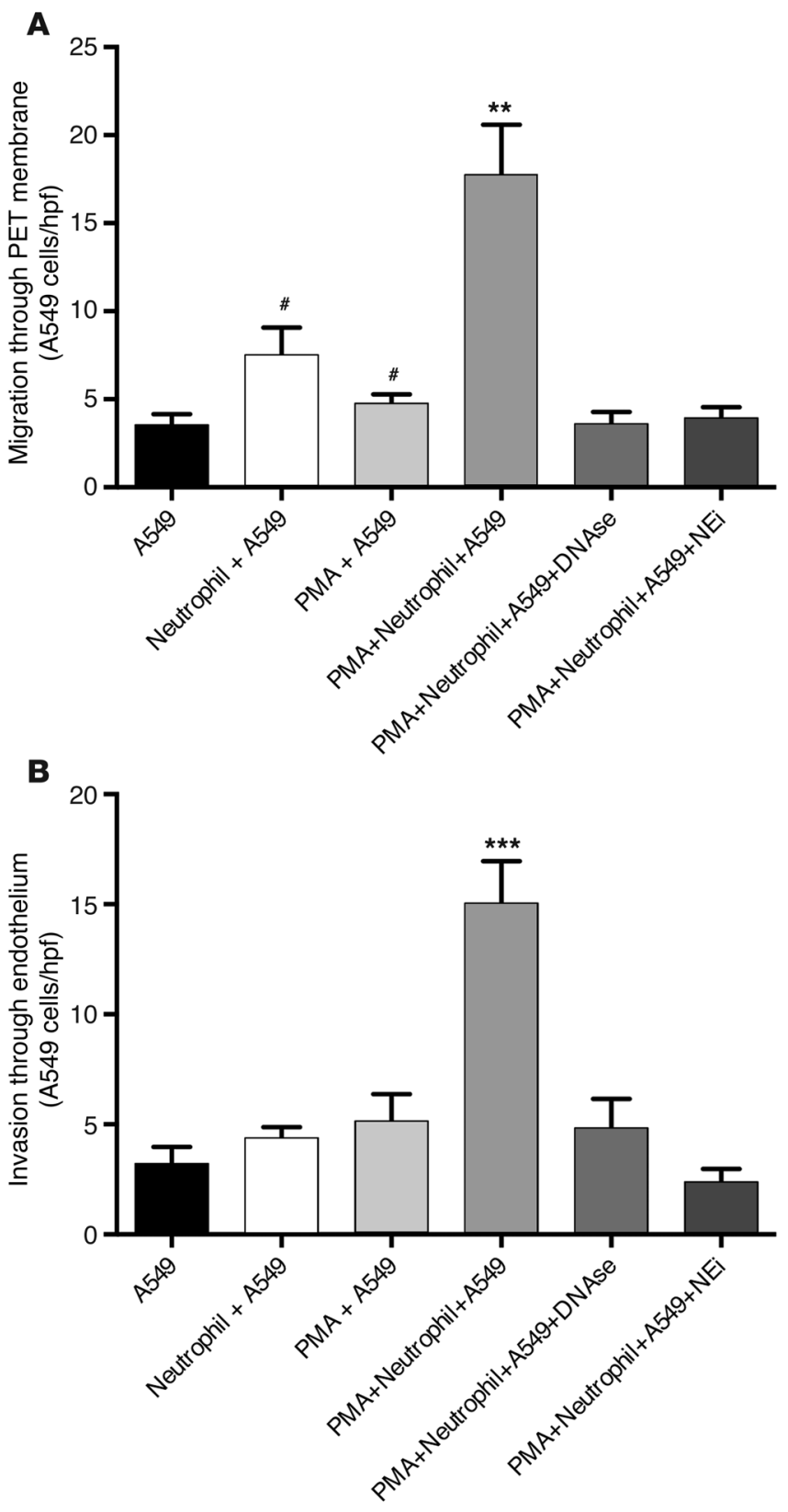

liver at 24 and 48 hours was observed compared with that in mice after sham surgery (mean 2 cells per hpf at 24 hours and 1.5 cells per hpf at 48 hours; $P=\mathrm{NS}$ ) (Figure 6C).

NETs stimulate migration and invasion of buman lung carcinoma cell lines in vitro. The demonstration that entrapment within NETs is associated with tumor cell adhesion and subsequent micrometastasis and macrometastasis formation implies that NETs may stimulate migration and invasion. We sought to test this directly in vitro (Figure 7). In the presence of neutrophils alone, a slight but nonsignificant increase in migration of A549 cells through PET membranes was appreciated compared with A549 cells alone (mean 3.6 cells per hpf versus 7.5 cells per hpf; $P=\mathrm{NS}$ ) (Figure 7A). Addition of $25 \mathrm{nM}$ PMA resulted in a greater than 3-fold increase in migration (mean 17.8 cells per hpf; $P<0.01$ versus neutrophil plus A549) (Figure 7A). However, addition of PMA to A549 cells in the absence

\section{Figure 7}

Migration and invasion of A549 cells in vitro is promoted in the presence of intact NETs. In the presence of neutrophils exposed to PMA, (A) A549 cell migration through 8- $\mu \mathrm{m}$ PET membranes and (B) invasion through endothelial monolayers was increased compared with A549 cells alone, A549 cells in the presence of unstimulated neutrophils, and A549 cells stimulated with PMA ( $25 \mathrm{nM})$ in the absence of neutrophils. This phenotype was reversed back to control levels after addition of DNAse $1(1,000 \mathrm{U})$ or pretreatment of neutrophils with NEi $(5 \mu \mathrm{m})$. Data are presented as mean \pm SEM from $n=2-4$ separate experiments. ${ }^{* \star} P<0.01,{ }^{* \star *} P<0.001$ versus $A 549$ alone. ${ }^{\#} P<0.05$ versus $A 549$ plus neutrophils and PMA. Significance was determined using 1-way ANOVA with post-hoc multiple comparisons with Tukey's HSD post-hoc analysis.

of neutrophils failed to induce migration to the same extent (mean 4.8 cells per hpf versus A549 alone; $P=$ NS) (Figure 7A). Migration of A549 cells in the presence of PMA and neutrophils was abrogated by the addition of 1,000 U DNAse 1 or pretreatment of neutrophils with $5 \mu \mathrm{M}$ NEi (mean 3.6 and 3.9 cells per hpf, respectively, versus A549 alone; $P=$ NS) (Figure 7A).

Similarly, in the presence of neutrophils alone, there was no appreciable increase in invasion of A549 cells through endothelial monolayers and PET membranes compared with A549 cells alone (mean 4.4 versus 3.3 cells per hpf; $P=\mathrm{NS}$ ) (Figure 7B). Conversely, addition of $25 \mathrm{nM}$ PMA resulted in a greater than 4 -fold increase in invasion (mean 15.1 cells per hpf versus control; $P<0.001$ ) (Figure 7B). However, addition of PMA to A549 cells in the absence of neutrophils failed to induce invasion at levels above control (mean 5.2 cells per hpf; $P$ = NS versus control) (Figure 7B). Invasion of A549 cells in the presence of PMA and neutrophils was abrogated by the addition of 1,000 U DNAse 1 or pretreatment of neutrophils with $5 \mu \mathrm{M} \mathrm{NEi}$ (mean 4.8 and 2.4 cells per hpf, respectively; $P=$ NS versus control) (Figure 7B). Taken together, these results support the role of intact NETs as the agents responsible for enhanced tumor cell migration and invasion in vitro.

\section{Discussion}

Surgical resection of solid tumors confers improved survival in appropriately selected patients. However, patients who suffer severe postoperative infections are more likely to die of metastasis than those who do not $(20,22,24,25)$. Mitigating the impact of severe infectious complications is not feasible in current clinical practice, in part because of the limited understanding of the biological processes linking infection and tumor progression. Clinical and experimental evidence suggests that activated neutrophils may facilitate metastatic progression in the context of systemic infection $(26,30,33,34)$. Accordingly, therapeutic strategies aimed at modifying tumor neutrophil interactions may one day maximize the therapeutic benefit of surgery in this important cohort of patients.

NETs are formed in response to infectious stimuli and constitute a recently described addition to the antimicrobial armamentarium of neutrophils (41). They have been observed to trap pathogens in vitro and in vivo $(44,46,48)$. In this study, we provide evidence suggesting that NETs are able to trap CTCs in an analogous manner in the context of severe postoperative sepsis, thus promoting early adhesion of tumor cells to distant organ sites.

Severe postoperative infections are characterized by a sustained disseminated polymicrobial infection with representative flora derived from the skin or operative site (55). For example, following gastrointestinal surgery complicated by leakage of enteric 
contents, patients may manifest clinical sequelae ranging from intra-abdominal abscess to fulminant peritonitis, with cultured organism reflecting an intestinal origin. Because neoplastic disease progression in humans appears to be influenced predominantly by severe, as opposed to any septic, complication, selecting an experimental model that reproduces these features was considered imperative. CLP closely approximates the severe septic complications that can occur after a number of gastrointestinal surgeries, including colorectal, gastric, and esophageal oncologic resections $(13,20,56)$. By definition, CLP results in the rapid onset of polymicrobial sepsis with endogenous flora. Furthermore, CLP has been validated as a robust and reproducible septic stimulus $(51,52,57,58)$. Consequently, it was selected in lieu of experimental models of sepsis relying on a single microorganism or bacterial component (e.g., LPS).

CLP-induced sepsis resulted in widespread microvascular DNA deposition, which stained positive for NE- and NET-associated histones. This phenotype was abolished after neutrophil depletion and exogenous administration of DNAse 1 and NEi, implicating NETs as the likely source of extracellular DNA. The finding of widespread NET deposition following CLP is not unexpected, and extensive data in the literature exists that describes NET formation in vivo in the context of sepsis $(48,59,60)$.

In this study, we demonstrated that NET formation in vivo after CLP is susceptible to exogenous pharmacologic inhibition with DNAse and NEi. Both DNAse and inhibition of NE have been shown to abrogate NET formation both in vitro and in vivo $(48,53,59,61-63)$. DNAse is thought to act via degradation of the DNA structure of NETs (48). Meng et al. demonstrated decreased circulating levels of DNA after administration of DNAse to mice subjected to CLP. This was associated with increased 24-hour mortality from uncontrolled infection, which was attributed to NET degradation and consequent bacterial dissemination (59). This finding is supported by the demonstration that the virulence of Group A Streptococcus in vivo is directly related to bacterial DNAse expression (64). Along these lines, exogenous administration of DNAse has been shown to eliminate intravascular neutrophil-derived DNA and histone in a murine model of gram-negative sepsis (48). Finally, thrombotic complications arising as a result of NET formation in murine models of malignancy can be attenuated via DNAse administration. In vitro, we and others have shown that addition of DNAse to appropriately stimulated neutrophils abolishes extracellular DNA deposition (ref. 53 and Supplemental Figure 6).

NE is abundantly expressed within NETs and is involved in their formation $(41,45,47,53)$. Through its enzymatic activity on histones, NE acts to promote chromatin decondensation and is required for DNA extrusion during NET formation. Indeed, mice lacking NE have been shown to be incapable of forming NETs. The NEi used in this study has been reported previously to inhibit NET formation in vitro. We corroborated this finding in addition to demonstrating an inhibitory role for NET formation in vivo (Supplemental Figures 4 and 6).

The gross metastatic data presented imply that CLP favors tumor progression and metastasis. Because CLP results in widespread NET deposition, and because metastasis formation is decreased after both DNAse and NEi administration, a possible role for NETs in tumor progression after infection arises. In vivo, NETs are thought to function as a bacterial trapping mechanism, preventing bacterial dissemination and uncontrolled infection
$(48,59)$. Along these lines, we postulate that under septic conditions, NETs may act to trap CTCs, thereby favoring adhesion of neoplastic cells in distant organ sites.

Early adhesive events after tumor dissemination are an important precursor to the development of metastatic disease $(3,30,35,65)$. Consistent with our hypothesis that NETs are able to trap CTCs, we visualized tumor cell arrest within areas of histone staining, which was consistent with that in NETs adjacent to neutrophils (48). These findings were consistent across cell lines and host metastatic organs (liver and lung), suggesting that this novel metastatic mechanism is widely applicable to various cancer sites and target organs.

Sepsis in vivo is associated with the deposition of cytotoxic histones, which promote microvascular coagulation. The possibility that tumor cell trapping in this context is due to passive retention thus arises. Video analysis demonstrates unimpeded trafficking of neutrophils through sinusoidal spaces after tumor cell retention. In addition, red blood cells were visualized passing through vascular spaces, which were stained densely for NETs. These observations argue against passive tumor cell retention as the sole adhesive mechanism. While a detailed examination is beyond the scope of this study, these observations raise the possibility that NET-mediated tumor cell trapping is specific.

Neutrophils support early adhesion through a number of mechanisms $(35,65)$. We have previously demonstrated that neutrophil depletion abrogates both early adhesion of malignant cells within the liver and the development of microscopic and macroscopic hepatic metastases (30). However, our study is the first to implicate NETs as a novel neutrophil-dependent adhesive mechanism. This is highlighted by the fact that hepatic tumor cell arrest in neutrophil-depleted mice is greatly enhanced if tumor cell administration is preceded by reinfusion of neutrophils stimulated with PMA. At the doses used in this study, PMA has been shown to result in NET formation by neutrophils $(41,53)$. This phenotype is reversed by presumed NET disruption with either DNAse 1 or NEi prior to neutrophil reinfusion. Because mice in all groups differed only in the manner in which the reinfused neutrophils were treated, the observed phenotype can more confidently be attributed to the adhesive action of NETs on tumor cells rather than the systemic effects of inflammation or the pharmacologic agents used.

In order to further support an adhesive interaction between NETs and neoplastic cells, an in vitro adhesion assay comprising only neutrophils and either murine (H59) or human (A549) lung cancer cell lines was used. Stimulation of neutrophils with PMA produced a significant increase in tumor cell adhesion compared with that of controls. This adhesion was robust and persisted at physiologically relevant shear stresses. However, as in the in vivo experiments, this was abolished by the addition of DNAse 1 or pretreatment of neutrophils with NEi. This effect was identical in assays using both human and murine cancer cells, arguing against a cell-specific phenomenon.

Confocal imaging in vitro demonstrated clusters of cells embedded within DNA webs adjacent to but not in direct contact with neutrophils. This finding was corroborated by scanning electron microscopy images demonstrating intimate contact between NETs and adherent tumor cells.

After adhesion in distant organ sites, tumor cells must be able to survive and grow in order to form metastases (31). In this study, adhesion in vivo was persistent, with mice subjected to CLP exhibiting significantly greater numbers of hepatic micrometastases at 24 and 48 hours compared with controls. In keeping with the 
results thus far, this phenotype was abolished by treatment with DNAse or NEi after CLP, again implicating NETs as players in this process. The demonstration that trapped tumor cells are able to survive and ultimately go on to form gross metastatic nodules contrasts with reports demonstrating that NETs are cytotoxic to epithelial and endothelial cells $(49,66)$. Treatment of endothelial and epithelial cells with isolated NETs resulted in $40 \%$ to $80 \%$ cytotoxicity. However, cell death was not complete, with a substantial proportion $(20 \%)$ remaining viable and able to form colonies. Furthermore, NET disruption with DNAse 1 or NEi failed to rescue cells from these cytotoxic effects, which were attributed predominantly to histones (49).

With regard to our study, it appears that some trapped tumor cells do survive NET sequestration. In addition, because our interventions (DNAse 1 and NEi) only affect NET quantity but not cytotoxicity, the differences in the numbers of micrometastases observed likely reflect differences in initial adhesion and not cell viability.

In order for adherent cells to effectively grow in distant organ sites, migration and invasion through endothelium is required $(31,67)$. The in vivo data presented suggest that NETs may facilitate these processes during sepsis, as adherent cells ultimately go on to form micrometastases and macrometastases. This hypothesis is supported by the in vitro demonstration of increased migration and invasion of A549 lung cancer cells in the presence of intact NETs.

These results are in keeping with reports that NET-associated proteins are involved in tumor progression. For example, NE is active in elaborated NETs and has been shown to promote extracellular matrix degradation and tumor cell shedding, thereby favoring the development of metastasis (67). These laboratory observations have a clinical corollary, with elevated serum levels of $\mathrm{NE}$ conferring a poor prognosis in patients with lung cancer (67). Along these lines, NETs contain high concentrations of MMP-9. This enzyme is associated with increased cell migration, invasion, tumor dissemination, and metastasis $(41,68,69)$.

In conclusion, this study is the first to implicate NETs as potential facilitators of tumor progression in the context of postoperative sepsis. We directly visualized tumor cell trapping within NETs in murine liver and lung in real time. This was associated with a drastic increase in hepatic metastatic disease burden compared with nonseptic controls. Furthermore, we demonstrated that NET formation in vivo can be inhibited by exogenous administration of DNAse 1 and NEi with favorable oncologic outcomes in this experimental model. This raises the possibility that mitigating the adverse oncologic consequences of severe sepsis in patients with cancer is at least plausible. While additional studies are required to further validate these results, we propose that the NET represents a novel potential therapeutic target in selected patients with cancer.

\section{Methods}

\section{Cells culture and cell lines}

The A549 human lung carcinoma cell line was purchased from Cedarlane. Murine Lewis lung carcinoma cell subline H59, which stably expresses GFP after plasmid transfection, was obtained and cultured as previously described (19). HUVECs were purchased from Cell Systems. Cells were maintained in DMEM F12 containing 10\% FBS, RPMI containing 10\% FBS, and DMEM containing $20 \% \mathrm{FBS}$, respectively, and incubated at $37^{\circ} \mathrm{C}$, $5 \% \mathrm{CO}_{2}$. Human neutrophils were isolated from healthy subjects using
Ficoll Hypaque density centrifugation as previously described (70). Only isolates with $>98 \%$ purity and viability, as determined by Turk's staining and Trypan Blue exclusion, were used.

\section{Animals}

Seven- to ten-week-old C57BL/6 mice (25 g; Charles River) were used for all experiments. Peritonitis was induced by CLP as previously described $(57,58)$. Briefly, a midline laparotomy was performed under inhaled anesthesia, and the cecum was exteriorized. The distal $1 \mathrm{~cm}$ of the cecum was ligated with a 4-0 silk suture, and the tip of the cecum was perforated on the antimesenteric aspect with a 25 -gauge needle. The cecum was returned to the abdominal cavity, and the abdomen was closed in 2 layers with a 4-0 silk suture. Animals received $0.2 \mathrm{mg} / \mathrm{kg}$ buprenorphine subcutaneously every 12 to 24 hours or as required. Sham animals had their cecums exteriorized without CLP. Mice were monitored postoperatively for signs of systemic sepsis, including changes in posture or activity, abdominal tenderness, rubor, and calor as described previously (58). Mice were treated with daily administration of DNAse $1(2.5 \mathrm{mg} / \mathrm{kg}$ [2,000 U/mg] intramuscularly Roche) or NEi GW311616A (2.2 mg/kg by gavage; Sigma-Aldrich), starting 24 hours prior to CLP until the termination of the experiment. These agents were selected given their demonstrated safety profile in previous animal and human experiments $(32,71,72)$.

For PMN depletion, mice received intraperitoneal injection of PMN-depleting antibodies (150 $\mu$ g anti-GR1 RB6-8C5; Cedarlane) as previously described (73). For PMN reinfusion experiments, neutrophils were isolated from bone marrow of syngeneic mice as described by Mocsai et al. (74). Briefly, tibias and femurs were removed and stripped of their muscles. The bone marrow was flushed using Hanks' balanced salt solution supplemented with $0.5 \% \mathrm{FBS}$, and cell aggregates were disrupted via filtration through $70-\mu \mathrm{m}$ cell strainer (Falcon). Erythrocytes were removed by hypotonic lysis. Neutrophils were separated by density centrifugation over a Percoll (62\% vol/vol in HBSS; Sigma-Aldrich) gradient at $1,000 \mathrm{~g}$ for 30 minutes at $4{ }^{\circ} \mathrm{C}$. Neutrophils were recovered as a pellet at the bottom of the $62 \%$ Percoll and were $>90 \%$ pure and $95 \%$ viable as determined by methylene and Trypan blue staining. Incubation of isolated cells with FITC-conjugated anti-mouse Gr-1 (Ly6G) antibody ( $0.1 \mu \mathrm{g}$; Cedarlane) followed by analysis with a flow cytometer FACScan and analysis with CellQuest Software similarly demonstrated $>90 \%$ purity (Supplemental Figure 7).

For neutrophil reinfusion, $1 \times 10^{6}$ cells were injected into the spleen. In some experiments, isolated neutrophils were stimulated with $500 \mathrm{nM}$ PMA for 1 hour prior to reinfusion. Similarly, in some experiments, PMA stimulation took place in the presence of $10 \mu \mathrm{MNEi}$ or $1,000 \mathrm{U}$ DNAse 1 . Tumor cell injection (H59) followed neutrophil reinfusion after 20 minutes in all cases.

For intravital microscopy, mice were anesthetized with a mixture of $50 \mathrm{mg} / \mathrm{kg}$ ketamine (Wyeth Ayerst Canada) and $5 \mathrm{mg} / \mathrm{kg}$ xylazine (Bayer) injected intraperitoneally and maintained throughout the course of the experiments with doses via jugular vein cannula.

All experiments were performed in accordance with the guidelines of and with the approval of the McGill University Animal Care Committee.

\section{Gross metastasis assay}

Gross liver metastases were quantified 2 weeks after systemic tumor cell administration. All mice received intrasplenic injection of $3 \times 10^{4} \mathrm{H} 59$ cells followed by splenectomy on postoperative day 2 after CLP or sham surgery. Mice were sacrificed 2 weeks later, and the livers were harvested in toto for analysis. Gross metastases were quantified with a maximum quantifiable number of 400 gross metastases per liver. Representative images were captured using a digital camera (Nikon). 


\section{Fluorescence intravital microscopy}

After induction of anesthesia, the right jugular vein and left carotid artery were cannulated for additional administration of anesthesia or reagents and tumor injection, respectively, as indicated. The anterior abdominal wall was removed from the costal margin to the midaxillary line bilaterally, and the falciform ligament was dissected away from the liver and gallbladder. In some experiments, the spleen was identified for intrasplenic tumor cell injection. The left lobe of the liver was gently positioned on a glass coverslip over the microscope objective. Animals were maintained at $37^{\circ} \mathrm{C}$ throughout the experiment with a heating pad or heated stage.

For epifluorescence microscopy, mice were visualized with an inverted microscope equipped with a $\times 20$ objective and digital camera for image acquisition (Nikon TE300). For SD-IVM, the exposed liver or lung lobe was visualized with an Olympus IX81 microscope equipped with a confocal light path (Wave-Fx; Quorum) based on a modified Yokogawa CSU-10 head (Yokogawa Electric Corporation) using a UPLANSAPO $\times 10 / 0.40$ or UPLANSAPO $\times 20 / 0.70$ air objectives. Three laser excitation wavelengths $(488,561$, and $635 \mathrm{~nm}$; Cobalt) were used in rapid succession and visualized with the appropriate long-pass filters (Semrock). Exposure times for excitation wavelengths were $303 \mathrm{~ms}$ (488 nm), $303 \mathrm{~ms}(561 \mathrm{~nm})$, and $300 \mathrm{~ms}$ (635 nm). A back-thinned EMCCD $512 \times 512$ pixel camera (C9100-13, Hamamatsu) was used for fluorescence detection. Volocity acquisition software (Improvision) was used to drive the microscope.

\section{Visualization and quantification of NETs}

NETs were visualized by fluorescence imaging of their components. Extracellular DNA was labeled with Sytox Green $(5 \mu \mathrm{M})$, histone H2AX was labeled with Alexa Fluor 555 anti-mouse H2AX antibody (5 $\mu \mathrm{g})$ (Cedarlane), and NE was labeled with Alexa Fluor 555 anti-mouse NE antibody $(0.6 \mu \mathrm{g})$ (Santa Cruz Biotechnology Inc.). Neutrophils were visualized with E-fluor 660 anti-mouse Gr-1 (Ly6G) antibody (3 $\mu \mathrm{g})$ (Cedarlane). Endothelium was imaged with PE anti-mouse PECAM-1 antibody $(3 \mu \mathrm{g})$. All antibodies and dyes were visualized 15 minutes after intravenous administration.

NETs were quantified within hepatic and pulmonary vasculature. Briefly, images were acquired as z-stacks (1- $\mu \mathrm{m}$ intervals) and saved as extended focus images in tiff format. Images in the green channel were exported to Image (NIH) for analysis. Contrast was adjusted in each image to minimize background and autofluorescence in order to try and minimize the variation in background fluorescence between experiments. A minimum brightness threshold was then set to yield only positive staining, and the same threshold and contrast settings were applied to each image within a given experiment. Threshold images were then converted to binary black-and-white images, and the area of positive staining was calculated as described previously (48). Data are expressed as relative area of fluorescence compared with sham surgery.

\section{In vivo adhesion assays}

Sinusoidal arrest. Ten minutes following intrasplenic injection of $5 \times 10^{4}$ H59-GFP cells or 48 hours following the intrasplenic injection of $1 \times 10^{6}$ H59-GFP cells into CLP, DNAse, NEi, and sham mice, the liver was visualized. GFP-expressing tumor cells were imaged using epifluorescence, and cells arrested within unoccluded sinusoids were considered adherent. Cells or tumor islands were quantified as the number of cells per micrometastatic foci in 8 to $10 \mathrm{hpf}$, and representative images were recorded.

Tumor cell entrapment within NETs. Neutrophils and H2AX were labeled in order to visualize NETs, and instillation of $1 \times 10^{6} \mathrm{H} 59$ cells via carotid cannula was performed. Upon injection of tumor cells, continuous image acquisition over a period of 30 minutes was carried out. Following cessation of video acquisition, representative images were acquired as z-stacks for subsequent analysis.

\section{In vitro adhesion assays}

Static adhesion assays. $5 \times 10^{5}$ neutrophils were plated in 24-well tissue culture plates and allowed to incubate for 1 hour at $37^{\circ} \mathrm{C} 5 \% \mathrm{CO}_{2}$ in RPMI alone or RPMI containing $5 \mu \mathrm{M} \mathrm{NEi} .1 \times 10^{5} \mathrm{H} 59$-GFP cells were added to wells with 800 nM PMA or 800 nM PMA plus 1,000 U DNAse1. Unstimulated neutrophils and untreated tumor cells in RPMI served as controls. Following incubation for 4 hours at $37^{\circ} \mathrm{C} 5 \% \mathrm{CO}_{2}$, wells were washed with PBS and fixed in $4 \%$ PFA. Adhesion was quantified as the number of cells in 4 random hpf at $\times 20$ using a Nikon TE300 microscope.

\section{Neutrophil adhesion and quantification}

Neutrophils were quantified by prestaining with carboxyfluorescein succinimidyl ester (CFSE) (Cedarlane). $5 \times 10^{5}$ neutrophils were plated in 24-well tissue culture plates and allowed to incubate for 1 hour at $37^{\circ} \mathrm{C}$ $5 \% \mathrm{CO}_{2}$ in RPMI alone or RPMI containing $5 \mu \mathrm{M} \mathrm{NEi} .800 \mathrm{nM}$ PMA or 800 nM PMA plus 1,000 U DNAse 1 was subsequently added to wells. Following incubation for 4 hours at $37^{\circ} \mathrm{C} 5 \% \mathrm{CO}_{2}$, wells were washed with $\mathrm{PBS}$ and fixed in $4 \%$ PFA. Images in the green channel were exported to ImageJ (NIH) for analysis. Contrast was adjusted in each image to minimize background and autofluorescence in order to try and minimize the variation in background fluorescence between experiments. A minimum brightness threshold was then set to yield only positive staining, and the same threshold and contrast settings were applied to each image within a given experiment. Threshold images were then converted to binary black-and-white images, and the area of positive staining was calculated as described previously (48). Data are expressed as relative area of fluorescence compared with sham surgery.

\section{Dynamic adhesion assays}

Untreated neutrophils or neutrophils preincubated with $5 \mu \mathrm{M}$ NEi for 1 hour at $37^{\circ} \mathrm{C} 5 \% \mathrm{CO}_{2}$ were placed on $40-\mathrm{mm}$ coverslips and allowed to adhere at $37^{\circ} \mathrm{C} 5 \% \mathrm{CO}_{2}$ for 1 hour. Concurrently, solutions of $5 \times 10^{5}$ cells/ml H59 cells were prepared in RPMI alone, RPMI plus $800 \mathrm{nM}$ PMA, or RPMI plus $800 \mathrm{nM}$ PMA and 1,000 U DNAse. After incubation, 40-mm coverslips with above treated neutrophils were placed in a parallel flow chamber (Bioptechs). Cell suspensions were perfused over neutrophil monolayers for 10 minutes at shear rates of 1 dyne $/ \mathrm{cm} / \mathrm{s}^{-1}$. To quantify adhesion, coverslips were fixed with $4 \%$ PFA for 10 minutes, washed with PBS, and mounted on glass slides. Cells were quantified in 5 to $10 \mathrm{hpf}$.

For video acquisition, neutrophils were stained with E-fluor 660 antimouse Gr-1 antibody ( $3 \mu \mathrm{g})$ for 1 hour at $37^{\circ} \mathrm{C}, 5 \% \mathrm{CO}_{2}$. Neutrophils were plated on $40-\mathrm{mm}$ glass coverslips as above. Concurrently, tumor cell perfusates were created as describe above with the addition of $20 \mathrm{mM}$ Sytox orange to the solution. Cell suspensions were perfused over neutrophil monolayers as described above. Cell interactions under flow conditions were visualized using an Olympus IX81 microscope equipped with a confocal light path (Wave-Fx; Quorum) (Calgary Camera) at $\times 10$ magnification.

\section{Confocal microscopy}

NET formation at the termination of static adhesion was confirmed using confocal microscopy. Neutrophils were plated on $13-\mathrm{mm}$ coverslips (Fisher Scientific) and treated as in the static adhesion assay. Neutrophils were stained with CellTracker Red (Invitrogen), A549 cells were stained with CellTracker Orange (Invitrogen), and extracellular DNA was stained with Sytox Green (Molecular Probes). Representative images were captured using a Zeiss LSM 780 laser scanning confocal microscope using a $\times 40 / 1.20 \mathrm{~W}$ corr C-Apochromat air objective. Three laser excitation wavelengths $(514,543$, and $633 \mathrm{~nm}$; Argon, DPSS, $\mathrm{HeNe}$ ) were used. 


\section{Scanning electron microscopy}

Neutrophils were plated on 13-mm coverslips (Fisher Scientific) and treated as in the static adhesion assay. Cells were fixed in $2.5 \%$ glutaraldehyde and then post-fixed using repeat incubations with $1 \%$ osmium tetroxide $/ 1 \%$ tannic acid. Samples were dehydrated with graded increasing concentrations of ethanol, critical point dried using a Leica EM CPD030 critical point drying device (Leica Microsystems, Austria), and coated with $5 \mathrm{~nm}$ carbon. Imaging was done with the Hitachi S-4700 FE-SEM.

\section{Functional assays}

Twenty-four-well Boyden chambers with 5- $\mu \mathrm{m}$ PET membranes (Fisher Scientific) were used for migration and invasion assays. Invasion assays differed in that HUVECs were placed in the upper chamber and allowed to proliferate for 72 hours After 72 hours, monolayer integrity was confirmed by verifying impermeability of the upper chamber to culture medium. $2.5 \times 10^{5}$ untreated neutrophils or neutrophils pretreated with $5 \mu \mathrm{M}$ NEi for 1 hour were placed in the upper chamber. A549 cells from $80 \%$ confluent cultures were detached using $0.5 \%$ trypsin (Wisent). Cells were adjusted to a concentration of $2.5 \times 10^{6} \mathrm{cells} / \mathrm{ml}$ and stained for 10 minutes with CFSE (Molecular Probes). $2.5 \times 10^{5}$ cells were added to the upper wells. Granulocyte-tumor cell suspensions were treated with 25 nM PMA alone, 25 nM PMA plus 1,000 U DNAse 1, or media alone. Isolated suspensions of A549 and A549 plus 25 nM PMA served as controls. The cell suspensions were incubated for 24 hours at $37^{\circ} \mathrm{C} 5 \% \mathrm{CO}_{2}$. After incubation, the contents of the upper chambers were aspirated, washed with PBS, and wiped with a sterile cotton swab. Cells adherent to the undersurface of the membrane were quantified in 4 random hpf, and representative images were taken as described above. NET formation was confirmed by epifluorescence microscopy at the termination of the experiments by epifluorescence microscopy.

\section{Statistics}

One-way ANOVA with post-hoc Tukey honestly significant difference (HSD) analysis for multiple comparisons was used. All data are presented as mean \pm SEM. Statistical significance was set at $P<0.05$. GraphPad Prism 6 software was used for all statistical analysis and graphing.

\section{Acknowledgments}

Scanning electron microscopy would not have been possible without the assistance of Facility for Electron Microscopy Research at McGill University. Samples were prepared by Jeannie Mui and Line Mongeon. Significant assistance with confocal microscopy was provided by the McGill University Health Center research institute core imaging facility at the Royal Victoria Hospital. In particular, we would like to thank Min Fu for her assistance. GFP-tagged H59 cells were a gift from the laboratory of Pnina Brodt.

Received for publication October 23, 2012, and accepted in revised form May 2, 2013.

Address correspondence to: Lorenzo Ferri, Department of Surgery, McGill University, 1650 Cedar Ave. - L9-112, Montreal General Hospital, Montreal, Quebec, Canada, H3G 1A4. Phone: 514.934.1934, ext. 44327; Fax: 514.934.4432; E-mail: lorenzo.ferri@mcgill.ca.
1. Parkin DM, Bray F, Ferlay J, Pisani P. Global cancer statistics, 2002. CA Cancer J Clin. 2005; 55(2):74-108.

2. Mountain CF. Revisions in the international system for staging lung cancer. Chest. 1997; 111(6):1710-1717.

3. McDonald B, Spicer J, Giannais B, Fallavollita L, Brodt $P$, Ferri LE. Systemic inflammation increases cancer cell adhesion to hepatic sinusoids by neutrophil mediated mechanisms. Int J Cancer. 2009; 125(6):1298-1305.

4. van Hagen $P$, et al. Preoperative chemoradiotherapy for esophageal or junctional cancer. $N$ Engl J Med. 2012;366(22):2074-2084.

5. Khan O, Goh S, Byrne B, Somers S, Mercer S, Toh S. Long-term outcomes of extended proximal gastrectomy for oesophagogastric junctional tumours. World J Surg. 2011;35(10):2245-2251.

6. Gonzalez-Neira A. Pharmacogenetics of chemotherapy efficacy in breast cancer. Pharmacogenomics. 2012;13(6):677-690.

7. Sinha VR. Critical aspects in rationale design of fluorouracil-based adjuvant therapies for the management of colon cancer. Crit Rev Ther Drug Carrier Syst. 2012;29(2):89-148.

8. Shapiro M. The role of adjuvant chemotherapy in early-stage and locally advanced non-small cell lung cancer. Cleve Clin J Med. 2012;1:eS42-eS45.

9. Sawabata $\mathrm{N}$, et al. Circulating tumor cells in peripheral blood caused by surgical manipulation of non-small-cell lung cancer: pilot study using an immunocytology method. Gen Thorac Cardiovasc Surg. 2007;55(5):189-192.

10. Shaw C, et al. Palliative venting gastrostomy in patients with malignant bowel obstruction and ascites. Ann Surg Oncol. 2013;20(2):497-505.

11. Wood DE, Liu YH, Vallières E, Karmy-Jones R, Mulligan MS. Airway stenting for malignant and benign tracheobronchial stenosis. Ann Thorac Surg. 2003; 76(1):167-172.

12. Van Den Berg YW, Reitsma PH. Not exclusively tissue factor: neutrophil extracellular traps provide another link between chemotherapy and thrombosis. JThromb Haemost. 2011;9(11):2311-2312.

13. Tsou CC, et al. Risk factors and management of anastomotic leakage after radical gastrectomy for gastric cancer. Hepatogastroenterology. 2011; 58(105):218-223.

14. Schussler O, et al. Postoperative pneumonia after major lung resection. Am J Respir Crit Care Med. 2006; 173(10):1161-1169.

15. Pierie JP, de Graaf PW, van Vroonhoven TJ, Obertop $\mathrm{H}$. Healing of the cervical esophagogastrostomy. J Am Coll Surg. 1999;188(4):448-454.

16. Smith RL, et al. Wound infection after elective colorectal resection. Ann Surg. 2004;239(5):599-605.

17. Itani KM, Wilson SE, Awad SS, Jensen EH, Finn TS, Abramson MA. Ertapenem versus cefotetan prophylaxis in elective colorectal surgery. $N$ Engl J Med. 2006;355(25):2640-2651.

18. Siegel R, Naishadham D, Jemal A. Cancer statistics, 2013. CA Cancer J Clin. 2013;63(1):11-30.

19. Auguste P, Fallavollita L, Wang N, Burnier J, Bikfalvi A, Brodt P. The host inflammatory response promotes liver metastasis by increasing tumor cell arrest and extravasation. Am J Pathol. 2007; 170(5):1781-1792.

20. Lin JK, et al. The influence of fecal diversion and anastomotic leakage on survival after resection of rectal cancer. J Gastrointest Surg. 2011;15(12):2251-2261.

21. Ohtsuka $T$, et al. Infectious complications after gastric cancer surgery accelerate a rapid hepatic recurrence. Hepatogastroenterology. 2009; 56(94-95):1277-1280.

22. Matsuo K, et al. Significance of perioperative infection in survival of patients with ovarian cancer. Int J Gynecol Cancer. 2012;22(2):245-253.

23. Colotta F, Allavena P, Sica A, Garlanda C, Mantovani A. Cancer-related inflammation, the seventh hallmark of cancer: links to genetic instability. Carcinogenesis. 2009;30(7):1073-1081.

24. Farid SG, et al. Correlation between postoperative infective complications and long-term outcomes after hepatic resection for colorectal liver metastasis.
Ann Surg. 2010;251(1):91-100.

25. Andalib A, Ramana-Kumar AV, Bartlett G, Franco EL, Ferri LE. Influence of postoperative infectious complications on long-term survival of lung cancer patients: a population-based cohort study. J Thorac Oncol. 2013;8(5):554-561.

26. Teramukai S, et al. Pretreatment neutrophil count as an independent prognostic factor in advanced non-small-cell lung cancer: an analysis of Japan Multinational Trial Organisation LC00-03. Eur J Cancer. 2009;45(11):1950-1958.

27. De Larco JE, Wuertz BR, Furcht LT. The potential role of neutrophils in promoting the metastatic phenotype of tumors releasing interleukin-8. Clin Cancer Res. 2004;10(15):4895-4900.

28. Fridlender ZG, Albelda SM. Tumor-associated neutrophils: friend or foe? Carcinogenesis. 2012; 33(5):949-955.

29. Yamanaka T, Matsumoto S, Teramukai S, Ishiwata R, Nagai Y, Fukushima M. The baseline ratio of neutrophils to lymphocytes is associated with patient prognosis in advanced gastric cancer. Oncology. 2007;73(3-4):215-220.

30. Spicer JD, et al. Neutrophils promote liver metastasis via Mac-1-mediated interactions with circulating tumor cells. Cancer Res. 2012;72(16):3919-3927.

31. Huh SJ, Liang S, Sharma A, Dong C, Robertson GP. Transiently entrapped circulating tumor cells interact with neutrophils to facilitate lung metastasis development. Cancer Res. 2010; 70(14):6071-6082.

32. Phillipson M, Kubes P. The neutrophil in vascular inflammation. Nat Med. 2011;17(11):1381-1390.

33. Lee YY, et al. Pretreatment neutrophil:lymphocyte ratio as a prognostic factor in cervical carcinoma. Anticancer Res. 2012;32(4):1555-1561.

34. Gondo T, et al. Prognostic value of neutrophil-to-lymphocyte ratio and establishment of novel preoperative risk stratification model in bladder cancer patients treated with radical cystectomy. Urology. 2012;79(5):1085-1091.

35. Slattery MJ, Dong C. Neutrophils influence mela- 
noma adhesion and migration under flow conditions. Int J Cancer. 2003;106(5):713-722.

36. Liang $S$, et al. Two-dimensional kinetics of beta 2 -integrin and ICAM-1 bindings between neutrophils and melanoma cells in a shear flow. Am J Physiol Cell Physiol. 2008;294(3):C743-C753.

37. Liang S, Hoskins M, Khanna P, Kunz RF, Dong C. Effects of the tumor-leukocyte microenvironment on melanoma-neutrophil adhesion to the endothelium in a shear flow. Cell Mol Bioeng. 2008 ; 1(2-3):189-200.

38. Houghton AM, et al. Neutrophil elastase-mediated degradation of IRS-1 accelerates lung tumor growth. Nat Med. 2010;16(2):219-223.

39. Coussens LM, Tinkle CL, Hanahan D, Werb Z. MMP-9 supplied by bone marrow-derived cells contributes to skin carcinogenesis. Cell. 2000; 103(3):481-490.

40. Chen HC, et al. Neutrophil elastase induces IL-8 synthesis by lung epithelial cells via the mitogen-activated protein kinase pathway. J Biomed Sci. 2004; 11(1):49-58.

41. Brinkmann V, et al. Neutrophil extracellular traps kill bacteria. Science. 2004;303(5663):1532-1535.

42. Fuchs TA, et al. Novel cell death program leads to neutrophil extracellular traps. J Cell Biol. 2007; 176(2):231-241.

43. Clark SR, et al. Platelet TLR4 activates neutrophil extracellular traps to ensnare bacteria in septic blood. Nat Med. 2007;13(4):463-469.

44. Pilsczek FH, et al. A novel mechanism of rapid nuclear neutrophil extracellular trap formation in response to Staphylococcus aureus. JImmunol. 2010; 185(12):7413-7425.

45. Metzler KD, et al. Myeloperoxidase is required for neutrophil extracellular trap formation: implications for innate immunity. Blood. 2011;117(3):953-959.

46. Urban CF, et al. Neutrophil extracellular traps contain calprotectin, a cytosolic protein complex involved in host defense against Candida albicans. PLoS Pathog. 2009;5(10):e1000639.

47. Guimaraes-Costa AB, et al. Leishmania amazonensis promastigotes induce and are killed by neutrophil extracellular traps. Proc Natl Acad Sci U S A. 2009;106(16):6748-6753

48. McDonald B, Urrutia R, Yipp BG, Jenne CN, Kubes P. Intravascular neutrophil extracellular traps capture bacteria from the bloodstream during sepsis.
Cell Host Microbe. 2012;12(3):324-333.

49. Saffarzadeh M, et al. Neutrophil extracellular traps directly induce epithelial and endothelial cell death: a predominant role of histones. PLoS One. 2012; $7(2): e 32366$

50. Villanueva E, et al. Netting neutrophils induce endothelial damage, infiltrate tissues, and expose immunostimulatory molecules in systemic lupus erythematosus. J Immunol. 2011; 187(1):538-552.

51. Toscano MG, Ganea D, Gamero AM. Cecal ligation puncture procedure. JVis Exp. 2011;(51):2860.

52. Hubbard WJ, et al. Cecal ligation and puncture. Shock. 2005;24(suppl 1):52-57.

53. Papayannopoulos V, Metzler KD, Hakkim A, Zychlinsky A. Neutrophil elastase and myeloperoxidase regulate the formation of neutrophil extracellular traps. J Cell Biol. 2010;191(3):677-691.

54. Munafo DB, Johnson JL, Brzezinska AA, Ellis BA, Wood MR, Catz SD. DNase I inhibits a late phase of reactive oxygen species production in neutrophils. J Innate Immun. 2009;1(6):527-542.

55. Alexander JW, Solomkin JS, Edwards MJ. Updated recommendations for control of surgical site infections. Ann Surg. 2011;253(6):1082-1093.

56 . Blencowe NS, et al. Reporting of short-term clinical outcomes after esophagectomy: a systematic review. Ann Surg. 2012;255(4):658-666.

57. Gommerman JL, et al. A role for CD21/CD35 and CD19 in responses to acute septic peritonitis: a potential mechanism for mast cell activation. J Immunol. 2000;165(12):6915-6921.

58. Cuenca AG, Delano MJ, Kelly-Scumpia KM, Moldawer LL, Efron PA. Cecal ligation and puncture. Curr Protoc Immunol Chapter. 2010;19:Unit 19.13.

59. Meng W, et al. Depletion of neutrophil extracellular traps in vivo results in hypersusceptibility to polymicrobial sepsis in mice. Crit Care. 2012;16(4):R137.

60. LiY, et al. Identification of citrullinated histone $\mathrm{H} 3$ as a potential serum protein biomarker in a lethal model of lipopolysaccharide-induced shock. Surgery. 2011;150(3):442-451

61. Papayannopoulos V, Staab D, Zychlinsky A. Neutrophil elastase enhances sputum solubilization in cystic fibrosis patients receiving DNase therapy. PLoS One. 2011;6(12):e28526.

62. Jovanovic B, Anastasova L, Rowe EW, Palic D. Hydroxylated fullerenes inhibit neutrophil func- tion in fathead minnow (Pimephales promelas Rafinesque, 1820). Aquat Toxicol. 2011;101(2):474-482.

63. Demers $M$, et al. Cancers predispose neutrophils to release extracellular DNA traps that contribute to cancer-associated thrombosis. Proc Natl Acad SciUS A. 2012;109(32):13076-13081.

64. Buchanan JT, et al. DNase expression allows the pathogen group A Streptococcus to escape killing in neutrophil extracellular traps. Curr Biol. 2006; 16(4):396-400.

65. Zhang P, Ozdemir T, Chung CY, Robertson GP, Dong C. Sequential binding of alphaVbeta 3 and ICAM-1 determines fibrin-mediated melanoma capture and stable adhesion to CD11b/CD18 on neutrophils. J Immunol. 2011;186(1):242-254.

66. Gupta AK, et al. Activated endothelial cells induce neutrophil extracellular traps and are susceptible to NETosis-mediated cell death. FEBS Lett. 2010; 584(14):3193-3197.

67. Piccard H, Muschel RJ, Opdenakker G. On the dual roles and polarized phenotypes of neutrophils in tumor development and progression. Crit Rev Oncol Hematol. 2012;82(3):296-309.

68. Pinilla S, et al. Tissue resident stem cells produce CCL5 under the influence of cancer cells and thereby promote breast cancer cell invasion. Cancer Lett. 2009;284(1):80-85.

69. Dufour A, Sampson NS, Zucker S, Cao J. Role of the hemopexin domain of matrix metalloproteinases in cell migration. J Cell Physiol. 2008;217(3):643-651.

70. Nauseef WM. Isolation of human neutrophils from venous blood. Methods Mol Biol. 2007;412:15-20.

71. Macdonald SJ, et al. The discovery of a potent, intracellular, orally bioavailable, long duration inhibitor of human neutrophil elastase--GW311616A a development candidate. Bioorg Med Chem Lett. 2001;11(7):895-898.

72. Ohbayashi H. Neutrophil elastase inhibitors as treatment for COPD. Expert Opin Investig Drugs. 2002; 11(7):965-980.

73. Bonder CS, Ajuebor MN, Zbytnuik LD, Kubes P, Swain MG. Essential role for neutrophil recruitment to the liver in concanavalin A-induced hepatitis. JImmunol. 2004;172(1):45-53.

74. Mocsai A, Zhang H, Jakus Z, Kitaura J, Kawakami T, Lowell CA. G-protein-coupled receptor signaling in Syk-deficient neutrophils and mast cells. Blood. 2003 ; 101(10):4155-4163. 